UDK 524.882

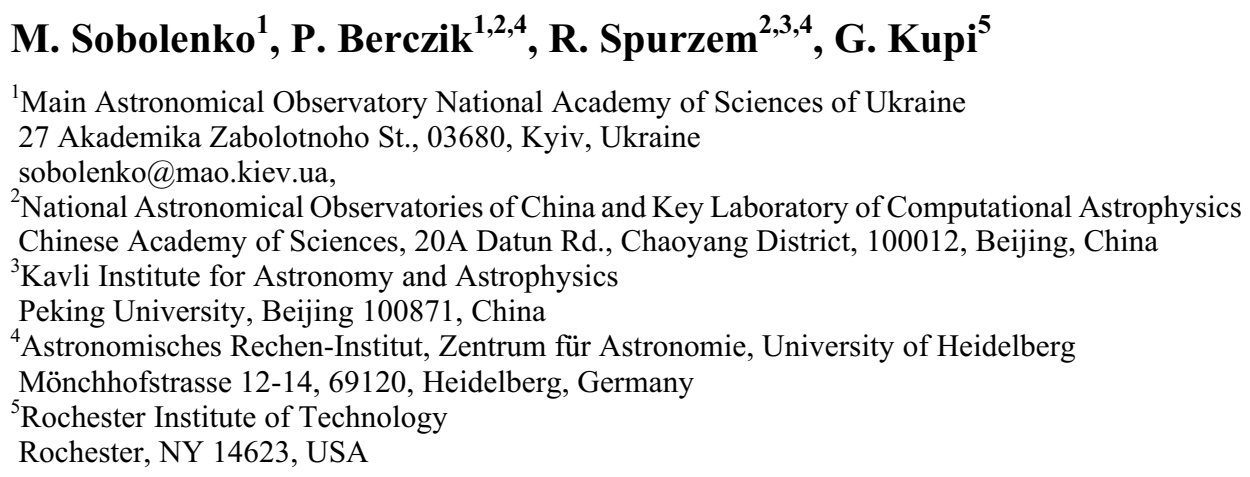

\title{
Fast coalescence of post-Newtonian Supermassive Black Hole Binaries in real galaxies
}

We present the results of theoretical modeling of supermassive black hole binary (SMBHB) mergers using direct 2 -body simulations with a Hermite integration scheme. The BH's gravitational interaction is described based

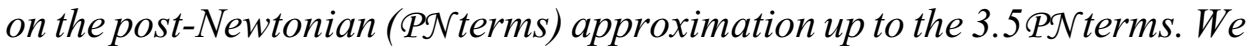
carry out a large set of runs using a parametric description of SMBHB orbits. The final time of the SMBHs gravitational coalescence is parametrized as a function of initial eccentricity $e_{0}$ and mass ratio $q$ of the binary. We carry out detailed tests of our coding. We tested our PS terms against the analytic prescription described at the theoretical works in middle $60^{\text {th }}$. The gravitational radiation polarization amplitudes $h_{+}$and $h_{\times}$from the SMBHBs merging process are also analyzed. Based on our numerical work we estimate the expected merging time for a list of selected potential SDSS SMBHBs. Our results show that the merging time is a strong function of the assumed initial eccentricities and fall within the range of thousands years.

ШВИДКЕ ЗЛИТТЯ ПОСТНЬЮТОНІВСЬКИХ ПОДВІЙНИХ НАДМАСИВНИХ ЧОРНИХ ДІР У РЕАЛЬНИХ ГАЛАКТИКАХ, Соболенко М. О., Бериик П. П., Шпурзем Р., Купі Г. - Приводяться результати теоретичного моделювання злиття подвійних надмасивних чорних дір за допомогою прямого 2-тільного моделювання з ермітівською схемою інтегрування. Гравітаційна взаємодія чорних дір описується постньютонівським наближенням до 3.5 PN-терму. На основі параметричного опису орбіт ПНЧД отримано великий набір моделей. 
Кінцевий час гравітаційного злиття ПНЧД параметризовано як функиію початкового ексиентриситету е 0 та відношення мас q подвійної. Проведено детальне тестування нашого коду. Ми порівнювали PN-mерми з аналітичним описом у теоретичних дослідженнях середини 1960-х рр. Проаналізовано амплітуду поляризованого гравітаиійного випромінювання $h_{+}$та $h_{\times}$nід час злиття ПНЧД. 3 використанням нашого числового коду очінено очікуваний час злиття для списку вибраних потениійних SDSS ПНЧД. Наші результати показують, шуо час злиття досягає тисяч років та є строгою функиією обраного початкового ексиентриситету.

БЫСТРОЕ СЛИЯНИЕ ПОСТНЬЮТОНОВСКИХ ДВОЙНЫХ СВЕРХМАССИВНЫХ ЧОРНЫХ ДЫР В РЕАЛЬНЫХ ГАЛАКТИКАХ, Соболенко М. А., Бериик П. П., Шпурзем Р., Купи Г. - Представлены результаты теоретического моделирования слияния двойных сверхмассивных чёрных дыр с помощью прямого 2-тельного моделирования с эрмитовской схемой интегрирования. Гравитационное взаимодействие чорных дыр описывается постньютоновским приближением до 3.5 PN-терма. На основе параметрического описания орбит ДСМЧД получен большой набор моделей. Конечное время гравитационного слияния ДСМЧД параметризовано как функиия начального эксиентрисета е ${ }_{0}$ и отношения масс q двойной. Проведено детальное тестирование намего кода. Сравнивались $\mathcal{P N}-$-термы с аналитическим описанием в теоретических исследованиях средины 1960-х г2. Проанализирована амплитуда поляризованного гравитационного излучения $h_{+}$и $h_{\times}$во время слияния ДСМЧД. С использованием нашего численного кода оченено ожидаемое время слияния для списка выбранных потенциальных SDSS ДСМЧД. Наши результаты показывают, что время слияния достигает тысяч лет и является строгой функиией выбранного начального эксиентриситета.

\section{INTRODUCTION}

The formation and evolution of galaxies and their SMBHs are connected in several ways. This relation can be found already at the early phases of protogalaxies formation [64], also at the later stages of hierarchical $\Lambda \mathrm{CDM}$ cosmology $[15,31,63]$ and also during the stages of different galaxy mergers $[36,42,51]$. One of the most simple and plausible channel of the SMBH mass growth is an accumulation of the BH's mass during host-galaxy mergers. Gas accretion can significantly increase the mass of BHs during "wet" merging that triggers star formation [3, 14, 25, 45, 57, 61]. Stellar accretion can also increase BH masses even in "dry" merging during the formation the giant elliptical galaxies $[5,44,46,67,71]$. The $M-\sigma$ relation, that shows a connection between the mass of the SMBH and the mass of the central bulge of their host galaxies [30], we assume is evidence for such a sce- 
nario. The fact that the distribution of the most luminous and massive active galactic nuclei peaks at higher redshifts also support this idea [34]. SMBHBs inside merging galaxies could be one of the most powerful sources of gravitational waves $(\mathrm{GW})$, which can be detected by the Pulsar Timing Array (PTA) or future space-based missions, such as LISA/eLISA, DESIGO/BBO [1, 33, 69]. The dynamical evolution of SMBHBs in the center of a merged stellar system can be traditionally divided in three phases [4].

(I) Two BHs can form a pair inside the merging host galaxy due to dynamical friction in the stellar background. Then these components sink into the centre of the stellar distribution. SMBHBs start to be "hard" when the length of the semimajor axis of the binary reaches the value:

$$
a \leq a_{h} \equiv \frac{G \mu}{4 \sigma^{2}} \approx\left(\frac{2.7 \mathrm{pc}}{1+q}\right)\left(\frac{m_{2}}{10^{8} M_{\odot}}\right)\left(\frac{\sigma}{200 \mathrm{~km} / \mathrm{s}}\right)^{-2},
$$

where $G$ is a gravitational constant, mass of the BH's is $m_{2} \leq m_{1}$, mass ratio is $q=m_{2} / m_{1}, \mu=m_{1} m_{2} /\left(m_{1}+m_{2}\right)$ is a reduced mass, total mass is $M_{t o t}=$ $m_{1}+m_{2}$. This means that the binding energy per unit mass $|E| / M_{t o t}=G \mu / 2 a$ exceeds $\sim \sigma^{2}$ (the ambient stellar velocity dispersion) [50].

(II) Due to the slingshot interaction mechanism the binary can continue to harden via three-body scattering of single stars. If star's orbit intersects with the SMBHB orbit, a complex three-body interaction can eventually lead to the "ejection" of the star. This "ejected" star carries away energy and angular momentum from the binary (see references in $[49,68,70]$ ).

But if we assume spherical symmetry, the loss cone of the binary BH system can be depleted by the slingshot mechanism before this [2, 27]. Therefore the system hardening time can be more than the Hubble time [52]. This is the so called "final parsec problem" which can be solved in $\mathrm{N}$-body simulations assuming a more realistic stellar particle distribution in a rotating system $[6,39]$, oblate / triaxial potential $[29,38,60]$ or some combination of these configurations.

(III) At the third stage the components sink toward to the separation when GW emission begins to be efficient. Finally, the binary inspirals down to the coalescence, emitting a strong $\mathrm{GW}$ signal. For such a merger the two SMBHs have to reach a critical separation in a time shorter than the Hubble time (few Gyr):

$$
a_{G W} \approx 2 \times 10^{-3} f(e)^{1 / 4} \frac{q^{1 / 4}}{(1+q)^{1 / 2}}\left(\frac{M_{t o t}}{10^{6} M_{\odot}}\right)^{3 / 4} \mathrm{pc},
$$

where $f(e)=\left[1+(73 / 24) e^{2}+(37 / 96) e^{4}\right]\left(1-e^{2}\right)^{-7 / 2}$ is a function of the binary eccentricity $e[58,59]$.

To estimate the SMBHBs real merging times, we need to make our calculations with the real speed of light values. Such $N$-body simulations are already available in the literature (for example $[38,40]$ ). But on the real merging galaxy scale such simulations require a lot of computing resources. 
In this paper we propose a slightly different approach. We perform simulations for different sets of parameters with various "parametric" values of light speed (for example see [9]). To explore the connection between the real merging time $T_{m r g}$, the total mass $M_{\text {tot }}$ of the SMBHB and initial separation $R$ between the $\mathrm{BHs}$ we estimate a scaling between the merging time $T_{m r g}$ and the speed of the light $\mathrm{c}$ assuming the dependence between these parameters.

\section{NUMERICAL METHODS AND INITIAL CONDITIONS}

Some numerical details. For the two BHB dynamical orbit integration, we use the publicly available $\varphi \operatorname{GPU}^{*}[7,8]$ with a $4^{\text {th }}$ order Hermite integrator and block hierarchical individual time step scheme. This Hermite scheme requires us to know the acceleration and its first time-derivative, called jerk. Because we use this Hermite scheme for our PSN runs, we need to include the PSNcorrections also to the acceleration and jerk terms. In the $\varphi$ GPU code we use the generalized "Aarseth" type criteria for the time step definition [53]:

$$
\Delta t=\eta_{p}\left(\frac{A^{(1)}}{A^{(p-2)}}\right)^{1 /(p-3)},
$$

where

$$
A^{(k)}=\sqrt{\left|\mathbf{a}^{(k-1)} \| \mathbf{a}^{(k+1)}\right|+\left|\mathbf{a}^{(k)}\right|^{2}} .
$$

Here, $\mathbf{a}^{(k)}$ is the $k^{\text {th }}$ derivative of acceleration, $p$ is the order of the integrator, $\eta_{p}$ is the accuracy parameter. For a $4^{\text {th }}$-order Hermite scheme the timestep looks like:

$$
\Delta t=\eta_{4} \frac{A^{(1)}}{A^{(2)}}
$$

where

$$
A^{(1)}=\sqrt{\left|\mathbf{a}^{(0)} \| \mathbf{a}^{(2)}\right|+\left|\mathbf{a}^{(1)}\right|^{2}}, \quad A^{(2)}=\sqrt{\left|\mathbf{a}^{(1)} \| \mathbf{a}^{(3)}\right|+\left|\mathbf{a}^{(2)}\right|^{2}} .
$$

For all our runs we use the $\eta_{4}=0.018$ parameter.

Post-Newtonian formalism. We use a post-Newtonian formalism in the 2-body code for calculating the relativistic binary systems dynamics. The results for up to $2 \mathscr{P N}$ and even up to $2.5 \mathscr{P N}$ equations of binary motion in harmonic coordinates were obtained by Damour and Deruelle [17-20, 24]. For the $3 \mathcal{P N}$ and $3.5 \mathcal{P N}$ terms we can use two different ways of computation. One of the possibilities is to use the ADM-Hamiltonian formalism of general relativity $[22,54-56]$. Physically equivalent results $[21,23]$ can be obtained from the post-Newtonian iteration [11], when we compute the equation of motion directly (instead of via a Hamiltonian) in harmonic coordinates.

The equation of motion is a power series of $1 / c$, where $n$-PJN is proportional to $(v / c)^{2 n}$. Schematically, one can write the correction for acceleration during the motion of object in binary system as $[19,65]$ :

* ftp://ftp.mao.kiev.ua/pub/berczik/phi-GPU/ 
$\mathbf{a}_{\text {NoSpin }}=\mathbf{a}_{N}+\frac{1}{c^{2}} \mathbf{a}_{1 \mathscr{P N}}+\frac{1}{c^{4}} \mathbf{a}_{2 \mathscr{P N}}+\frac{1}{c^{5}} \mathbf{a}_{2.5 P \mathcal{N}}+\frac{1}{c^{6}} \mathbf{a}_{3 \mathscr{P N}}+\frac{1}{c^{7}} \mathbf{a}_{3.5 P \mathcal{N}}+O\left(\frac{1}{c^{8}}\right)$,

where $\mathbf{a}_{N}$ is the classical Newtonian acceleration; $\mathbf{a}_{1 \oplus \mathcal{N}}, \mathbf{a}_{2 \rho \mathcal{N}}, \mathbf{a}_{3 \rho \mathcal{N}}$ are the non dissipative terms which "conserve" the energy of the system. The $\mathbf{a}_{2.5 \mathscr{N}}, \mathbf{a}_{3.5 P \mathcal{N}}$ are the dissipative terms which "carry out" energy from the system due to GW emission. We apply all $\mathcal{P N}$ corrections up to order $O\left(1 / c^{8}\right)$, so the 3.5PSN correction is the highest order that we take into account. To compare our results with the analytical solution from classical articles $[58,59]$ we use the code just with the single $2.5 \mathcal{P N}$ term.

Similar to the equation of motion in the centre of mass frame [10] the acceleration for one particle can be written in the following form:

$$
\mathbf{a}_{\text {NoSpin }}=\frac{d \mathbf{v}}{d t}=-\frac{G M}{r^{2}}[(1+\mathcal{A}) \mathbf{n}+\mathscr{B} \mathbf{v}]
$$

where $r=|\mathbf{r}|$ is the separation between particles, $\mathbf{r}=\mathbf{r}_{1}-\mathbf{r}_{2}$ is the position of the particles, $\mathbf{n}=\mathbf{r} / r$ is the normalized relative position vector, $\mathbf{v}=\mathbf{v}_{1}-\mathbf{v}_{2}$ is the relative velocity. The functions $\mathcal{A}$ and $\mathcal{B}$ contain different orders of the $\mathcal{P N}$ approximation (similar to Eq. (7)).

For example the first $\mathcal{P N}$ correction term is given by:

$$
\begin{gathered}
A_{1 \oplus \mathcal{N}}=\left[\frac{5 G m_{1}}{r}+\frac{4 G m_{2}}{r}+\frac{3}{2}\left(\mathbf{n} \cdot \mathbf{v}_{2}\right)^{2}-\mathbf{v}_{1}^{2}+4\left(\mathbf{v}_{1} \cdot \mathbf{v}_{2}\right)-2 \mathbf{v}_{2}^{2}\right], \\
B_{1 \Re \mathcal{N}}=4\left(\mathbf{n} \cdot \mathbf{v}_{1}\right)-3\left(\mathbf{n} \cdot \mathbf{v}_{2}\right) .
\end{gathered}
$$

Detailed references and the complete description of the problem can be found in works such as $[9,10,43]$. The complete equations in post-Newtonian formalism up to 3.5PSN are given also in [10].

Adding the spin terms into the equation of motion we can describe as:

$$
\mathbf{a}_{\text {Spin }}=\mathbf{a}_{\text {NoSpin }}+\frac{1}{c^{3}} \mathbf{a}_{1.5 \mathscr{P N}, S O}+\frac{1}{c^{4}} \mathbf{a}_{2 \mathscr{P N}, S S}+\frac{1}{c^{5}} \mathbf{a}_{2.5 \mathcal{P N}, S O},
$$

where $\mathbf{a}_{1.5 P \mathcal{N}, S O}$ and $\mathbf{a}_{2.5 \mathcal{N}, S O}$ are the spin-orbit coupling terms, $\mathbf{a}_{2 \mathcal{P N}, S S}$ is the spin-spin coupling term (for example [26]). Now one can write the full equation (like Eq. (7)):

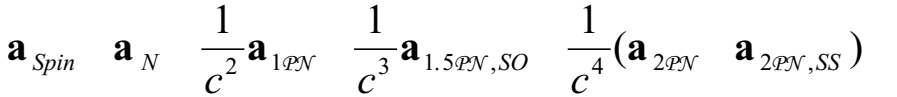

$$
\begin{aligned}
& +\frac{1}{c^{5}}\left(\mathbf{a}_{2.5 P \mathcal{N}}+\mathbf{a}_{2.5 P \mathcal{P N}, S O}\right)+\frac{1}{c^{6}} \mathbf{a}_{3 \mathscr{P N}}+\frac{1}{c^{7}} \mathbf{a}_{3.5 \mathscr{P N}}+O\left(\frac{1}{c^{8}}\right),
\end{aligned}
$$

where the full expression for $\mathbf{a}_{1.5 \mathrm{PN}, S O}$ and $\mathbf{a}_{2.5 \mathrm{PN}, S O}$ can be found in [26], for $\mathbf{a}_{2 \mathscr{N}, S S}$ can be found in [66]. The value of the physical spin is chosen from the the next expression:

$$
S^{\text {true }}=\chi \frac{G m^{2}}{c},
$$

where the value of $\chi$ is $[0,1]$. At the centre of the binary mass frame we have the $\operatorname{spin} \mathbf{S} \equiv \mathbf{S}_{1}+\mathbf{S}_{2}$. We use two body dynamics and spin-spin and spin-orbit 
coupling just for calculation of the first order of the gravitational waveform constraint (e.g. [41]):

$$
h^{i j} \approx \frac{4 G \mu}{D c^{4}}\left[v^{i} v^{j}-\frac{G M}{r} n^{i} n^{j}\right],
$$

where $Q^{i j}=2\left(v^{i} v^{j}-G M n^{i} n^{j} / r\right)$ is the usual quadrupole term (second time derivatives of the mass quadrupole moment tensor) and $D$ is the luminosity distance. Choosing the virtual detector orientation so that as the coordinate axes coincide with the source frame, we can describe the two-dimensional matrix with only two independent elements:

$$
h_{i j}=\left(\begin{array}{cc}
h_{+} & h_{\times} \\
h_{\times} & -h_{+}
\end{array}\right) .
$$

From $h_{i j}$ we can obtain the amplitude of polarization $h_{+}$and $h_{\times}[12,16$, 62].

Initial conditions and description of model. We assume that the two point masses which represents our BHs with masses $m_{1}$ and $m_{2}$ are placed at positions $Y_{1}$ and $Y_{2}$ on the $Y$ axis (see Fig. 1). For our analyses we choose the natural coordinate system of the two bodies, connected by the centre of mass of the system. The initial orbital velocity of the two point masses we chose so that the $X Y$ plane contains the full orbit. The initial separation between the components we defined as $R=\left|Y_{1}\right|+\left|Y_{2}\right|$. We also set the BH's mass ratio $q=m_{1} / m_{2}$. We assume that $m_{1} \leq m_{2}$. We also fix the total BH system mass $M_{t o t}=m_{1}+m_{2}$. The Keplerian motion of the two bodies can be fully

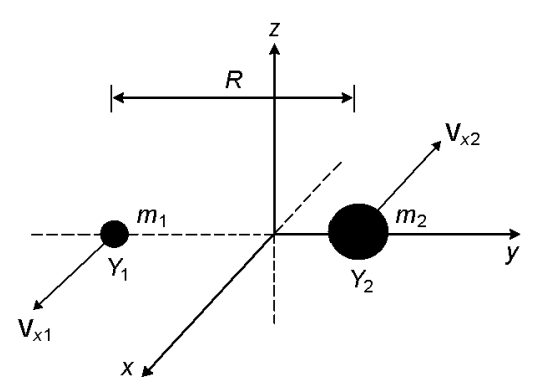

Fig. 1. Configuration of the system with two BHs

described by two main orbital parameters: the semimajor axis $a$ and eccentricity $e$. We can write the binding energy of the binary system:

$$
|E|=\frac{G m_{1} m_{2}}{2 a}=\frac{G \mu M_{t o t}}{2 a},
$$

where $\mu=m_{1} m_{2} / M_{\text {tot }}$ is the reduced mass. We also fix as a parameter the binary initial orbital eccentricity $e_{0}$. The initial setup of the particles we show in Figure 1. For further calculation we assume the normalization $R=1$ and $M_{\text {tot }}=m_{1}+m_{2}=1$. 
We use the N-body (NB) or called Hénon units [32] where we also accept $G=1$ and set the mass units $M$ and length units $R$ to unity ${ }^{*}$. Therefore the physical values of mass, length, energy, velocity and time will be in the form:

$$
\begin{gathered}
{[M]=M, \quad[L]=R,} \\
{[E]=\frac{G M^{2}}{R},} \\
{[V]=\left(\frac{G M}{R}\right)^{1 / 2}, \quad[T]=\left(\frac{R^{3}}{G M}\right)^{1 / 2} .}
\end{gathered}
$$

Consequently the light speed $c$ in $N$-body units is:

$$
c=\frac{c_{0}}{V}=c_{0}\left(\frac{G M}{R}\right)^{-1 / 2}=14213 \cdot\left(\frac{M}{10^{8} M_{\odot}}\right)^{-1 / 2}\left(\frac{R}{10^{3} \mathrm{pc}}\right)^{1 / 2},
$$

where $c_{0}$ is the light speed in physical units.

\section{DISCUSSION}

Scaling routine between merging time $T_{m r g}$ and "parametric" speed of the light was made for all models from Table 1 (for example see Fig. 2 for system with parameters $\left.M_{t o t}=1[\mathrm{NB}], q=0.5, R=1[\mathrm{NB}], e_{0}=0.25\right)$. Based on our post-Newtonian formalism (Eqs (7)-(12)) we can theoretically expect the relationship between merging time (which is directly proportional to the energy losses in our post-Newtonian formalism) and the light speed:

$$
T_{m r g 5} \propto b \cdot c^{5}, \quad T_{m r g 5+7} \propto d \cdot c^{5}+p \cdot c^{7},
$$

where $b, d$ and $p$ are the coefficients of the scaling. As we can see from Fig. 2 the difference between the two merging times are negligible. So, in this paper we use the $T_{m r g}$ as a basic approximation for the binary merging time $T_{m r g}$.

Table 1. The scale factor $b$ from Eq. (21) for various mass ratio $q$ and initial eccentricity $e_{0}$ (separation for each system $R=1[\mathrm{NB}]$ and total mass $M_{\text {tot }}=1[\mathrm{NB}]$ )

\begin{tabular}{c|c|c|c|c|c|c}
\hline \multirow{2}{*}{$e_{0}$} & \multicolumn{7}{c}{$b$} \\
\cline { 2 - 7 } & $q=1$ & $q=0.5$ & $q=0.333$ & $q=0.25$ & $q=0.2$ & $q=0.02$ \\
\hline 0.00 & $7.863 \mathrm{E}-02$ & $8.827 \mathrm{E}-02$ & $1.043 \mathrm{E}-01$ & $1.218 \mathrm{E}-01$ & $1.397 \mathrm{E}-01$ & $8.611 \mathrm{E}-01$ \\
0.25 & $2.578 \mathrm{E}-02$ & $2.900 \mathrm{E}-02$ & $3.437 \mathrm{E}-02$ & $4.027 \mathrm{E}-02$ & $4.639 \mathrm{E}-02$ & $3.375 \mathrm{E}-01$ \\
0.50 & $5.584 \mathrm{E}-03$ & $6.280 \mathrm{E}-03$ & $7.440 \mathrm{E}-03$ & $8.716 \mathrm{E}-03$ & $1.004 \mathrm{E}-02$ & $7.244 \mathrm{E}-02$ \\
0.75 & $4.648 \mathrm{E}-04$ & $5.225 \mathrm{E}-04$ & $6.186 \mathrm{E}-04$ & $7.243 \mathrm{E}-04$ & $8.339 \mathrm{E}-04$ & $6.003 \mathrm{E}-03$ \\
0.95 & $1.893 \mathrm{E}-06$ & $2.126 \mathrm{E}-06$ & $2.514 \mathrm{E}-06$ & $2.938 \mathrm{E}-06$ & $3.383 \mathrm{E}-06$ & $2.425 \mathrm{E}-05$ \\
0.99 & $8.146 \mathrm{E}-09$ & $9.123 \mathrm{E}-09$ & $1.076 \mathrm{E}-08$ & $1.255 \mathrm{E}-08$ & $1.441 \mathrm{E}-08$ & $1.023 \mathrm{E}-07$
\end{tabular}

\footnotetext{
http://en.wikipedia.org/wiki/N-body_units
} 


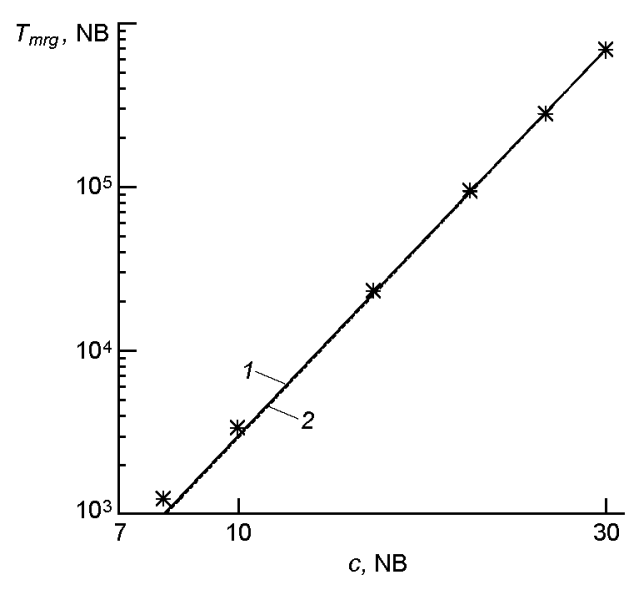

Fig. 2. Relation between the merging time $T_{m r g}$ and light speed $c$ for system from Table 1 (line $1-T_{m r g}=b c^{5}, 2-T_{m r g}=d c^{5}+p c^{7}$, stars simulation). Initial eccentricity $e_{0}=0.25$ and mass ratio $q=0.5$

We study the evolution of systems with various mass ratios and initial eccentricities, i. e. with various orbits. We use the following sets of the parameters: $q=1,0.5,0.333,0.25,0.2,0.02$ and $e=0.00,0.25,0.50,0.75$, $0.95,0.99$.

We apply the scaling factors from Table 1 to find the real merging times $T_{m r g}$ (in physical units) where the physical light speed is $c=2.99792458 \times$ $\times 10^{8} \mathrm{~m} / \mathrm{s}$.

We apply the above described "c-scaling procedure" for a wide range of physical parameters for masses $\left(10^{6} M_{\odot} \leq M_{\text {tot }} \leq 5 \cdot 10^{9} M_{\odot}\right)$ and the initial separation between the BHs $\left(10^{-3} \leq R \leq 10^{2} \mathrm{pc}\right)$. For each individual model we estimate the relation between the merging time $T_{m r g}$, separation between the BHs R and total mass $M_{\text {tot }}$ of the SMBHB (Fig. 3, 4). For example using Fig. 3 for system with $M_{\text {tot }}=10^{9} M_{\odot}, q=0.5, R=10$ pc, $e_{0}=0.5$ merging time $T_{\text {mrg }} \approx 1700$ years.

In a real cosmological merging scenario we expect that the SMBHBs merger does not evolve in isolation. High resolution cosmological numerical simulations (see references in $[28,37,48]$ ) show us that SMBHB mergers typically need to meet the next large galaxy in a time scale of 1-2 Gyr. If we assume the existence of a SMBH in this third galaxy too, in this case our binary $\mathrm{BH}$ is transformed to a triple $\mathrm{BH}$ system. Extensive direct $N$-body simulations of system with three BHs show that such a configuration is highly unstable $[1,13]$. So, we assume that if in a time scale of 1-2 Gyr our original BHB system does not merge, the possibility of such a merger becomes very unlikely. In Figures 3, 4 we show the 1 Gyr merging time as the solid black lines for the different initial eccentricities.

For some fixed time this relation can be written in the form:

$$
R=10^{\beta}\left(M_{S M B H B, 6}\right)^{3 / 4} \text {. }
$$

We found that with the rise of initial eccentricity $e_{0}$ the merging time $T_{m r g}$ of the system decreases. This behavior is valid for mass ratios from $q=$ $=1$ to $q=0.2$ and even for extremal $q=0.02$. The general conclusion from our set of runs is that the lower initial eccentricity (circular) orbits generally 


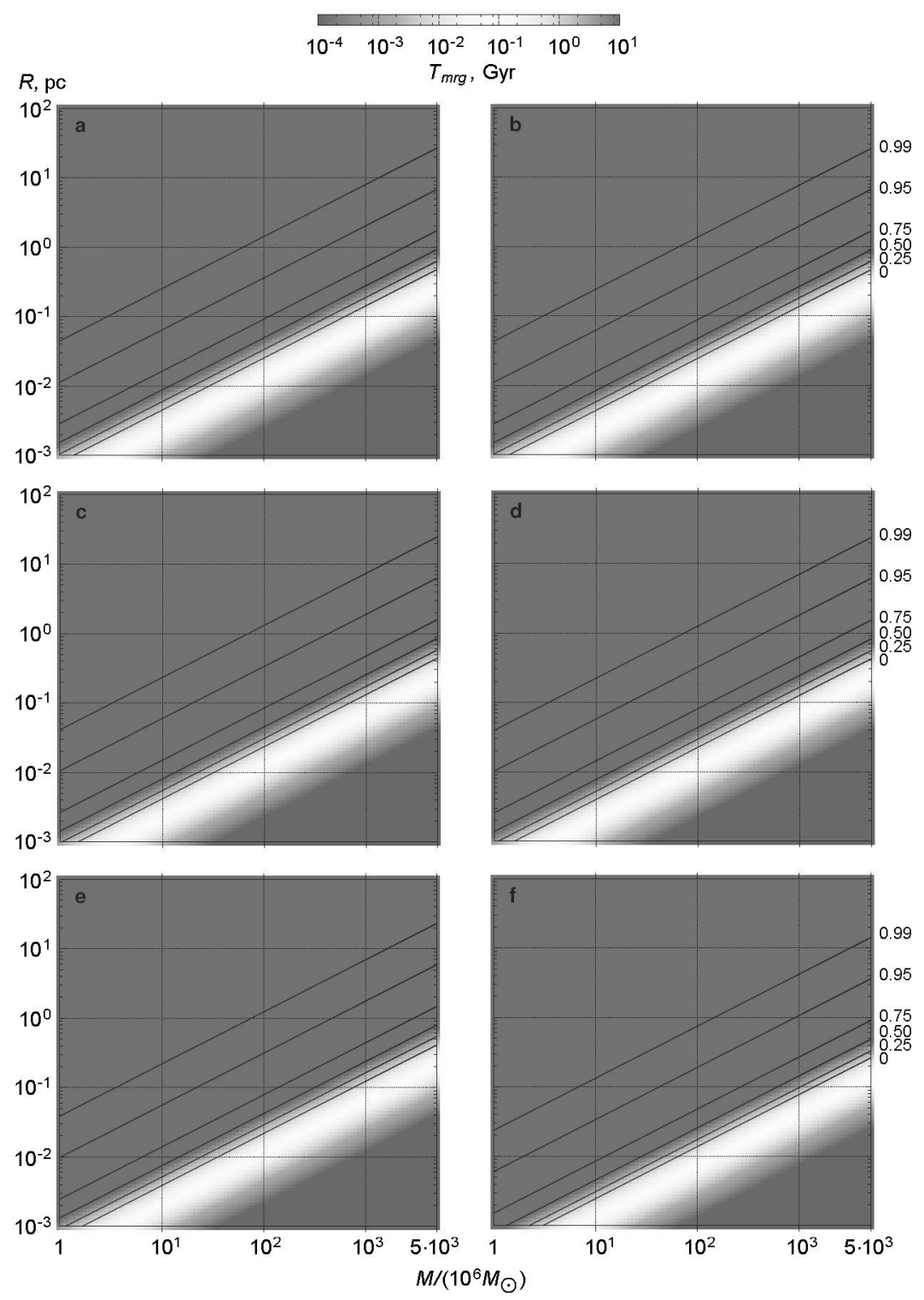

Fig. 3. The color coded final merging time $T_{m r g}$ of SMBHB as a function of total mass and initial separation of the binary. Each separate plot shows the merging time evolution for the specific mass ratio of the binary: $q=1(a), 0.5(b), 0.333(c), 0.25(d), 0.2(e), 0.02(f)$. On each plots we indicate the $1 \mathrm{Gyr}$ merging time line as a function of the initial eccentricity $e_{0}$ of the binary. Colored gamma for value $e_{0}=0.00$

have a longer merging time. For higher mass ratios even the eccentric orbits become more stable.

Comparison of the simulation results and theoretical work [58, 59] (which includes in the expressions only for the $2.5 \mathcal{P N}$ term) is shown in (Fig. 5, 6). For this numerical test we use the parameters $M_{t o t}=2[\mathrm{NB}], q=$ $=1, R=1[\mathrm{NB}], e_{0}=0.7, c=15[\mathrm{NB}]$ and we also include only the $2.5 \mathcal{P N}$ term. Our test simulations show that the numerical model behaves very similar to the theoretical curve. 


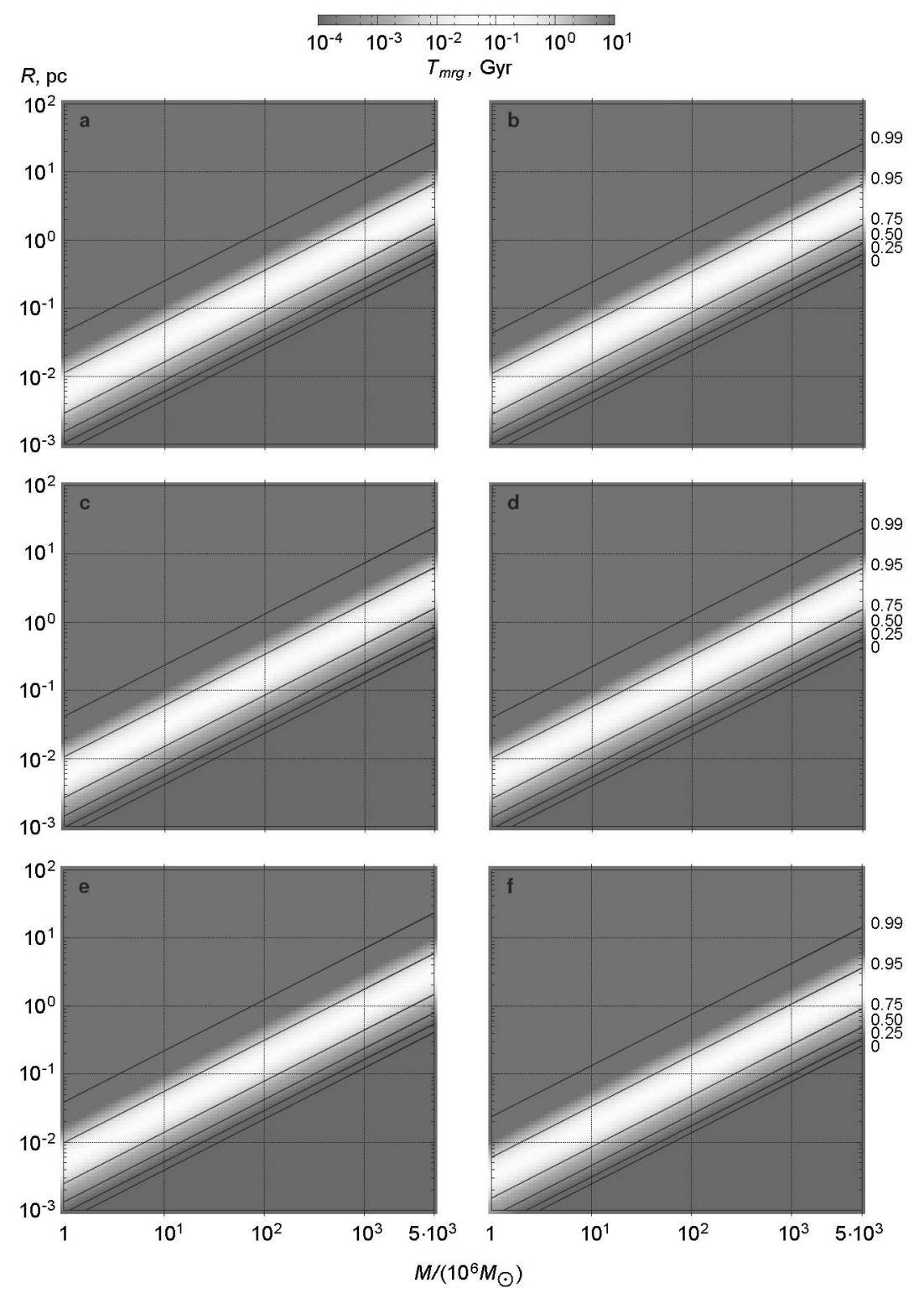

Fig. 4. The color coded final merging time $T_{m r g}$ of SMBHB as a function of total mass and initial separation of the binary. Each separate plot shows the merging time evolution for the specific mass ratio of the binary: $q=1(a), 0.5(b), 0.333(c), 0.25(d), 0.2(e), 0.02(f)$. On each plots we indicate the $1 \mathrm{Gyr}$ merging time line as a function of the initial eccentricity $e_{0}$ of the binary. Colored gamma for value $e_{0}=0.95$

For obtaining the GW constraints, for the selected test case $\left(M_{t o t}=\right.$ $\left.10^{8} M_{\odot}, q=0.5, R=0.01 \mathrm{pc}, e_{0}=0.95, \mathbf{S}_{1}=[0,0,1], \mathbf{S}_{2}=[0,0,1]\right)$, we use the spin-spin and spin-orbit coupling which was described above [12]. In Fig. 7 we show the first periastron passes for $h_{+}$and $h_{\times}$. In Fig. 8 we see the waveform during inspiraling just for $h_{+}$polarization (the $h_{\times}$looks similar). In Table 2 we present the GW frequencies for BHs with typical masses and binary system orbital parameters. 
Fig. 5. Comparison the simulation's evolution (dots) of the semimajor axis $a$ with analytical results (line) for a system with following initial parameters: $M_{\text {tot }}=2[\mathrm{NB}], q=1, R=1[\mathrm{NB}], e_{0}=$ $0.6, c=15[\mathrm{NB}]$ with just turning on $2.5 \mathcal{P N}$

Fig. 6. Comparison the simulation's evolution (dots) of the eccentricity $e$ with analytical results (line) for a system with following initial parameters: $M_{\text {tot }}=2[\mathrm{NB}], q=1, R=1[\mathrm{NB}], e_{0}=$ $0.6, c=15[\mathrm{NB}]$ with just turning on $2.5 \mathcal{P} \mathcal{N}$
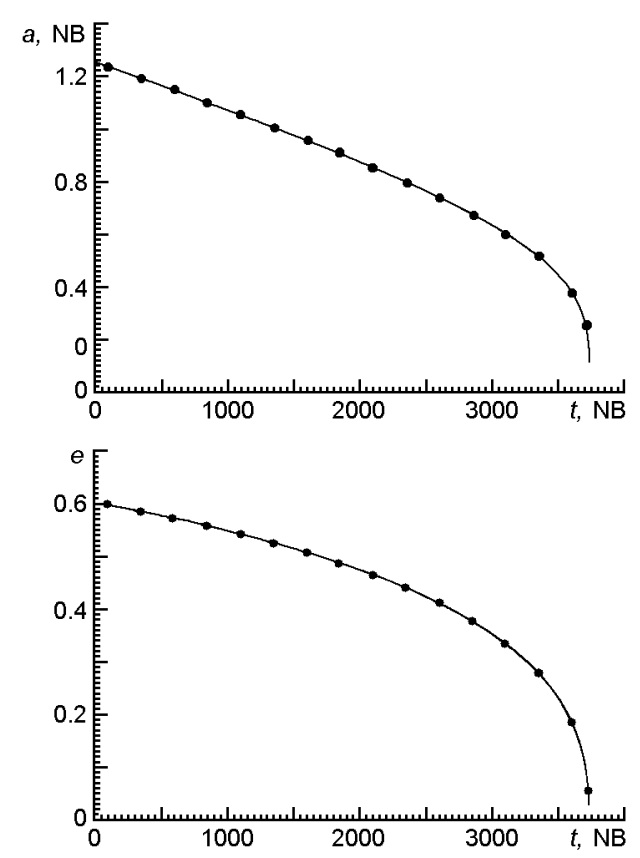

Table 2. The GW frequency for BHs with typical masses $M_{\text {tot }}$ and system parameters $q=0.5$, $e_{0}=0.95, R=0.01 \mathrm{pc}, S_{1}=[0,0,1], S_{2}=[0,0,1]$

\begin{tabular}{c|c|c|c|c}
$M_{\text {tot }} / M_{\odot}$ & $R\left(r_{S}\right)$ & $T_{\text {mrg }}, \mathrm{yr}$ & $T_{\text {orb }}, \mathrm{s}$ & $\nu, \mu \mathrm{Hz}$ \\
\hline $10^{9}$ & 104 & 0.6 & 866925 & 1.15 \\
$10^{8}$ & 1045 & 631.5 & 55175 & 18.1 \\
$10^{7}$ & 10451 & 655148.3 & 5933 & 169 \\
\hline
\end{tabular}

Table 3. Configurations of the systems for SDSS objects from [35] $(q=1)$

\begin{tabular}{c|c|c|c}
\hline SDSS ID & $z$ & $\log \left(M_{t o t} / M_{\odot}\right)$ & $r_{\max }, \mathrm{mpc}$ \\
\hline J075700.70+424814.5 & 1.17 & 9.1311 & 20 \\
J002444.11+003221.4 & 0.40 & 9.5618 & 102 \\
J004918.98+002609.4 & 1.94 & 9.3148 & 96 \\
J161609.50+434146.8 & 0.49 & 8.1696 & 21 \\
J093502.54+433110.7 & 0.46 & 9.3425 & 181 \\
J032223.02-000803.5 & 0.62 & 8.2827 & 32 \\
J095656.42+535023.2 & 0.61 & 8.2944 & 127 \\
\end{tabular}

Using our well tested $P \mathcal{N}$-routine we estimate the possible BHBs merging time for the set of SDSS objects [35]. The main parameters of the binary BHs we present in Table 3. We estimate binary $\mathrm{BH}$ expected merging times assuming different eccentricities $\left(e_{0}=0.00-0.99\right)$ of the orbits except $\mathrm{J} 1201$, for which we $e_{0}=0.3$. Also we calculated the merging time for the serendipitously discovered SDSS J120136.02+3000305.5 $(z=0.146)$ with system parameters $M_{\text {tot }}=1.08 \times 10^{7} M_{\odot}, q=0.08, r_{\max }=1.3 \mathrm{mpc}, e_{0}=0.3$ [47]. 

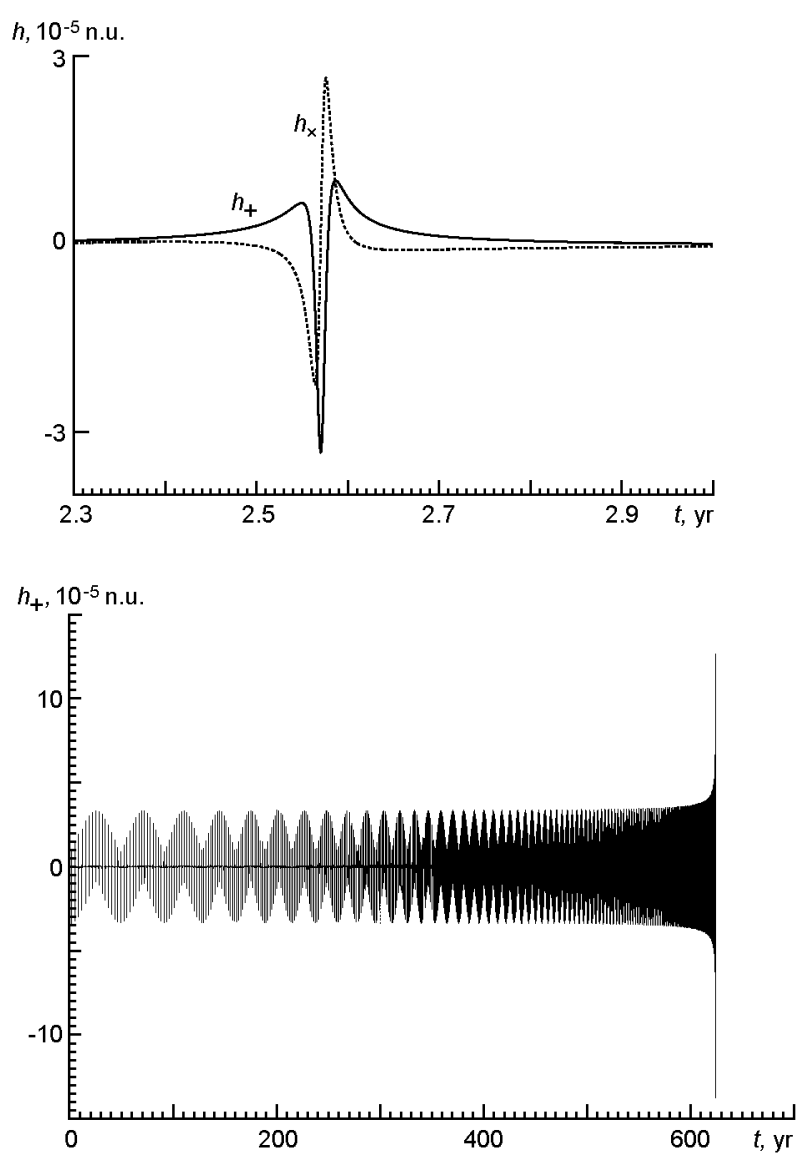

Fig. 7. Simulated strain from a $G W$ during the first periastron for a system with $M_{\text {tot }}=10^{8} M_{\odot}(q=$ $0.5), e_{0}=0.95, R=0.01 \mathrm{pc}, \dot{S}_{1}=[0$, $0,1], \mathbf{S}_{2}=[0,0,1]$
Fig. 8. Strain for a system with parameters $M_{\text {tot }}=10^{8} M_{\odot}(q=0.5)$, $e_{0}=0.95, R=0.01 \mathrm{pc}, \mathbf{S}_{1}=[0,0,1]$, $\mathbf{S}_{2}=[0,0,1]$

Table 4. Expected merging time $T_{m r g}$ for SMBHBs for the selected SDSS objects as the function of the eccentricities

\begin{tabular}{c|c|c|c|c|c|c|c}
\hline \multirow{2}{*}{$e_{0}$} & \multicolumn{7}{c}{$T_{m r g}, \mathrm{yr}$} \\
\cline { 2 - 9 } & $\mathrm{J} 0757$ & $\mathrm{~J} 0024$ & $\mathrm{~J} 0049$ & $\mathrm{~J} 1616$ & $\mathrm{~J} 0935$ & $\mathrm{~J} 0322$ & $\mathrm{~J} 0956$ \\
\hline 0.00 & $2.139 \mathrm{E}+04$ & $6.906 \mathrm{E}+05$ & $2.916 \mathrm{E}+06$ & $1.767 \mathrm{E}+07$ & $2.997 \mathrm{E}+07$ & $4.390 \mathrm{E}+07$ & $9.929 \mathrm{E}+09$ \\
0.25 & $6.796 \mathrm{E}+03$ & $2.213 \mathrm{E}+05$ & $9.385 \mathrm{E}+05$ & $5.714 \mathrm{E}+06$ & $9.672 \mathrm{E}+06$ & $1.420 \mathrm{E}+07$ & $3.214 \mathrm{E}+09$ \\
0.50 & $1.439 \mathrm{E}+03$ & $4.738 \mathrm{E}+04$ & $2.023 \mathrm{E}+05$ & $1.241 \mathrm{E}+06$ & $2.095 \mathrm{E}+06$ & $3.086 \mathrm{E}+06$ & $7.004 \mathrm{E}+08$ \\
0.75 & $1.221 \mathrm{E}+02$ & $3.873 \mathrm{E}+03$ & $1.668 \mathrm{E}+04$ & $1.040 \mathrm{E}+05$ & $1.745 \mathrm{E}+05$ & $2.591 \mathrm{E}+05$ & $5.920 \mathrm{E}+07$ \\
0.90 & $9.528 \mathrm{E}-01$ & $6.635 \mathrm{E}+00$ & $5.014 \mathrm{E}+02$ & $4.195 \mathrm{E}+02$ & $7.413 \mathrm{E}+02$ & $1.046 \mathrm{E}+03$ & $2.453 \mathrm{E}+05$ \\
0.99 & $9.255 \mathrm{E}-01$ & $6.435 \mathrm{E}+00$ & $7.780 \mathrm{E}+00$ & $2.960 \mathrm{E}+00$ & $1.946 \mathrm{E}+01$ & $4.893 \mathrm{E}+00$ & $1.007 \mathrm{E}+03$
\end{tabular}

As we can see from Table 4 some of the selected SDSS objects have a quite short merging time even for moderately large eccentricities $e_{0} \geq 0.75$. Almost all of the selected objects (except one J0956) have expected merging times only a few years for initial eccentricities $e_{0}=0$. However J1201 has an estimated $T_{m r g}=3.27 \mathrm{Myr}$, that is not such a gratifying result. Hopefully our merging time predictions can be tested with the larger SDSS4 observational catalogues, which are right now in a phase of observation. 


\section{CONCLUSION}

In our study we analyze the dynamical behavior of SMBHBs. We use a highly accurate direct 2-body code where we apply the additional $P \mathcal{N}$ terms up to 3.5 PSN for calculation of the gravitational forces which act on the BHs and spin-spin and spin-orbit coupling for calculation of GW constraints. As the main result we obtain the resulting merging time $T_{m r g}$ for a large set of initial mass ratios $q$ of the $\mathrm{BBH}$, initial masses, initial separations and orbital eccentricities $e_{0}$. This data we present as a set of color coded 3-D plots. We also make the original results presented on these plots for different mass ratios $q$ and initial eccentricities $e_{0}$ publicly available ${ }^{*}$. Our $\mathcal{P} \mathcal{N}$ treatment was extensively tested and the $\mathcal{P N}$ routines itself we also make publicly available via the same link above. In our high order direct 2-body implementation we use not only the $P \mathcal{N}$ accelerations but also the first derivatives of this accelerations. Our BHBs test calculations show that for $\mathrm{BH}$ masses in range $M_{t o t}=\left(10^{6}-10^{9}\right) M_{\odot}$ with a fixed initial separation $R=0.01 \mathrm{pc}$ and initial eccentricity $e_{0}=0.95$ the $\mathrm{GW}$ frequencies are well inside the

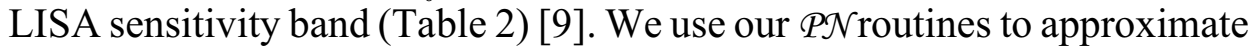
the expected merging time for the selected sample SDSS SMBHBs [35]. Our results show that for significantly large eccentricities the expected merging time for these objects are in the range of years.

\section{ACKNOWLEDGEMENTS}

MS acknowledge the financial support by the NASU under the Grant for young researchers. MS and PB acknowledge also the special support by the NASU under the Main Astronomical Observatory GRID/GPU computing cluster golowood project.

RS and PB acknowledge support by Chinese Academy of Sciences through the Silk Road Project at NAOC, through the "Qianren" special foreign experts program of China. MS gratefully acknowledges support for collaboration visits in Beijing under the same program.

Supported by the Strategic Priority Research Program "The Emergence of Cosmological Structures" of the Chinese Academy of Sciences, Grant N XDB09000000.

The main part of the simulations presented here was performed on the dedicated GPU clusters kepler at the ARI, funded under the grants I/80 041-043 and I/81 396 of the Volkswagen Foundation and the grants $823.219-439 / 30$ and /36 of the Ministry of Science, Research and the Arts of Baden-Württemberg, Germany.

The authors are acknowledges the support of the Volkswagen Foundation under the Trilateral Partnerships grant No. 90411.

Part of the code development work was conducted using the resources of the GPU cluster laohu at the Center of Information and Computing at the National Astronomical Observatories, Chinese Academy of Sciences,

ftp://ftp.mao.kiev.ua/pub/sobolenko/ 
funded by the Ministry of Finance of People's Republic of China under the grant ZDYZ2008-2.

We also acknowledge the helpful comments made by Stephen Justham.

1. Amaro-Seoane P., Sesana A., Hoffman L., et al. Triplets of supermassive black holes: astrophysics, gravitational waves and detection // Mon. Notic. Roy. Astron. Soc.2010.-402.-P. 2308-2320.

2. Amaro-Seoane P., Spurzem R. The loss-cone problem in dense nuclei // Mon. Notic. Roy. Astron. Soc.-2001.-327.-P. 995-1003.

3. Barton E. J., Geller M. J., Kenyon S. J. Tidally triggered star formation in close pairs of galaxies // Astrophys. J.-2000.-530.-P. 660-679.

4. Begelman M. C., Blandford R. D., Rees M. J. Massive black hole binaries in active galactic nuclei // Nature.-1980.-287.-P. 307-309.

5. Bell E. F., Naab T., McIntosh D. H., et al. Dry mergers in GEMS: The dynamical evolution of massive early-type galaxies // Astrophys. J.—2006. - 640. — P. 241-251.

6. Berczik P., Merritt D., Spurzem R., et al. Efficient merger of binary supermassive black holes in nonaxisymmetric galaxies // Astrophys. J.-2006. - 642.-P. 21-24.

7. Berczik P., Nitadori K., Zhong $S$., et al. High performance massively parallel direct N-body simulations on large GPU clusters // Inter. conf. "High Performance Computing”, Kyiv, Ukraine, October 8-10, 2011. - Kyiv, 2011.—P. 8-18.

8. Berczik P., Spurzem R., Wang L. Up to 700k GPU cores, Kepler, and the Exascale future for simulations of star clusters around black holes // Third International Conference "High Performance Computing" (HPC-UA 2013), Kyiv, Ukraine, October 7-17, 2013. - Kyiv, 2013.-P. 52-59.

9. Berentzen I., Preto M., Berczik P., et al. Binary black hole merger in galactic nuclei: Post-Newtonian simulations // Astrophys. J.—2009.-695.-P. 455-468.

10. Blanchet $L$. Gravitational radiation from post-Newtonian sources and inspiralling compact binaries // Liv. Revs Relativ.-2006.-9.-114 p.

11. Blanchet L., Faye G., Ponsot B. Gravitational field and equations of motion of compact binaries to 5/2 post-Newtonian order // Phys. Rev. D. - 1998. - 58, N 12. - 20 p.

12. Brem P. Effects of general relativity in direct N-body codes: Master's thesis. - Heidelberg: Astronomisches Rechen-Institut, 2011.-85 p.

13. Brem P., Amaro-Seoane P., Spurzem R. Relativistic mergers of compact binaries in clusters: the fingerprint of the spin // Mon. Notic. Roy. Astron. Soc._2013.-434. -P. 2999-3007.

14. Bridge C. R., Appleton P. N., Conselice C. J., et al. The role of galaxy interactions and mergers in star formation at $z=1.3$ : Mid-infrared properties in the Spitzer first look survey // Astrophys. J.-2007.—659.-P. 931—940.

15. Choi E., Naab T., Ostriker J. P., et al. Consequences of mechanical and radiative feedback from black holes in disc galaxy mergers // Mon. Notic. Roy. Astron. Soc.2014.-42.-P. 440-453.

16. Cutler $C$. Angular resolution of the LISA gravitational wave detector // Phys. Rev. D.-1998.-57.-P. 7089-7102.

17. Damour T. Gravitational radiation and the motion of compact bodies // Lecture Notes in Physics. — Berlin: Springer Verlag, 1983.-P. 59-144.

18. Damour T., Deruelle N. Generalized lagrangian of two point masses in the post-Newtonian approximation of general-relativity // Comptes rendus de l'Academie des Sci. Ser. II.-1981.-293.-P. 537-540.

19. Damour T., Deruelle N. Radiation reaction and angular momentum loss in small angle gravitational scattering // Phys. Lett. A.—1981. -87.— P.81—84.

20. Damour T., Deruelle N. The two-body problem and radiation damping in general- relativity // Comptes rendus de l'Academie des Sci. Ser. II._1982._294._P. 1355_ 
1357.

21. Damour T., Jaranowski P., Schäfer G. Equivalence between the ADM Hamiltonian and the harmonic-coordinates approaches to the third post-Newtonian dynamics of compact binaries // Phys. Rev. D.-2001.-63 (4).-11 p.

22. Damour T., Schäfer G. Lagrangians for n point masses at the second post-Newtonian approximation of general relativity // Gen. Relativity and Gravitation.-1985.17.-P. 879-905.

23. de Andrade V. C., Blanchet L., Faye G. Third post-Newtonian dynamics of compact binaries: Noetherian conserved quantities and equivalence between the harmonic coordinate and ADM-Hamiltonian formalisms // Classical and Quantum Gravity.2001.-18.-P. 753-778.

24. Deruelle N. Sur les équations du mouvement et le rayonnement gravitationnel d'un système binaire en Relativité Générale: PhD thesis. - Paris: Université Pierre et Marie Curie, 1982.

25. Di Matteo P., Bournaud F., Martig M., et al. On the frequency, intensity, and duration of starburst episodes triggered by galaxy interactions and mergers // Astron. and Astrophys.-2008.-492.-P. 31-49.

26. Faye G., Blanchet L., Buonanno A. Higher-order spin effects in the dynamics of compact binaries. I. Equations of motion // Phys. Rev. D. - 2006.-74, N 10.-19 p.

27. Frank J. Rees M. J. Effects of massive central black holes on dense stellar systems // Mon. Notic. Roy. Astron. Soc._1976.-176.-P. 633-647.

28. Governato F., Brook C., Mayer L., et al. Bulgeless dwarf galaxies and dark matter cores from supernova-driven outflows // Nature._2010._463.—P. 203-206.

29. Gualandris A., Merritt D. Long-term evolution of massive black hole binaries. IV. Mergers of galaxies with collisionally relaxed nuclei // Astrophys. J._-2012._ 744, N 1.-21 p.

30. Gültekin K., Richstone D. O., Gebhardt K., et al. The M-sigma and M-L relations in galactic bulges, and determinations of their intrinsic scatter // Astrophys. J._-2009._698.-P. 198-221.

31. Haehnelt M. G., Kauffmann G. Multiple supermassive black holes in galactic bulges // Mon. Notic. Roy. Astron. Soc.-2002._336.-P. 61-64.

32. Hénon M. H. The Monte Carlo method // Astrophys. and Space Sci._1971._14, N 1. -P. 151-167.

33. Hewitson M., Consortium eLISA. eLISA: A mission to study the entire universe with gravitational waves // Amer. Astron. Soc. Meeting Abstracts._2014._223.P. 248.

34. Hirschmann M., Somerville R. S., Naab T., et al. Origin of the antihierarchical growth of black holes // Mon. Notic. Roy. Astron. Soc._2012._426.-P. 237-257.

35. Ju W., Greene J. E., Rafikov R. R., et al. Search for supermassive black hole binaries in the Sloan Digital Sky Survey spectroscopic sample // Astrophys. J._2013._-777._16 p.

36. Kauffmann G., Haehnelt M. A unified model for the evolution of galaxies and quasars // Mon. Notic. Roy. Astron. Soc.-2000.-311.-P. 576-588.

37. Khan F. M., Berentzen I., Berczik P., et al. Formation and hardening of supermassive black hole binaries in minor mergers of disk galaxies // Astrophys. J.-2012.756. - $10 \mathrm{p}$.

38. Khan F. M., Holley-Bockelmann K., Berczik P., et al. Supermassive black hole binary evolution in axisymmetric galaxies: The final parsec problem is not a problem // Astrophys. J.-2013.-773.-6 p.

39. Khan F. M., Just A., Merritt D. Efficient merger of binary supermassive black holes in merging galaxies // Astrophys. J.-2011._-732.-8 p.

40. Khan F. M., Preto M., Berczik P., et al. Mergers of unequal-mass galaxies: super- 
massive black hole binary evolution and structure of merger remnants // Astrophys. J.-2012.-749.-14 p.

41. Kidder L. E. Coalescing binary systems of compact objects to (post) $)^{5 / 2}$-Newtonian order. V. Spin effects // Phys. Rev. D.-1995._52.-P. 821-847.

42. Komossa S. Observational evidence for supermassive black hole binaries // AIP Conf. Proc.-2003. - 686.-P. 161-174.- (The astrophysics of gravitational wave sources / Ed. J. M. Centrella).

43. Kupi G., Amaro-Seoane P., Spurzem R. Dynamics of compact object clusters: a postNewtonian study // Mon. Notic. Roy. Astron. Soc.—2006.-371.—P. 45-49.

44. Lin L., Cooper M. C., Jian H.-Y., et al. Where do wet, dry, and mixed galaxy mergers occur? A study of the environments of close galaxy pairs in the DEEP2 galaxy redshift survey // Astrophys. J.-2010.-718.-P. 1158-1170.

45. Lin L., Koo D. C., Weiner B. J., et al. AEGIS: Enhancement of dust-enshrouded star formation in close galaxy pairs and merging galaxies up to $z \sim 1 / /$ Astrophys. J.2007.-660.-P. 51-54.

46. Lin L., Patton D. R., Koo D. C., et al. The redshift evolution of wet, dry, and mixed galaxy mergers from close galaxy pairs in the DEEP2 galaxy redshift survey // Astrophys. J.-2008. - 681.-P. 232-243.

47. Liu F. K., Li S., Komossa S. A milliparsec supermassive black hole binary candidate in the galaxy SDSS J120136.02 + 300305.5 // Astrophys. J._2014._-786.-14 p.

48. Mayer L., Kazantzidis S., Escala A., et al. Direct formation of supermassive black holes via multi-scale gas inflows in galaxy mergers // Nature._-2010._466. - P. 1082_ 1084.

49. Merritt D. Brownian motion of a massive binary // Astrophys. J._2001. - 556. P. 245-264.

50. Merritt D., Milosavljević M. Massive black hole binary evolution // Liv. Revs Relativity.-2005.-8. - 63 p.

51. Milosavljević M., Merritt D. Formation of galactic nuclei // Astrophys. J.-2001.563.-P. 34-62.

52. Milosavljević M., Merritt D. The final parsec problem // AIP Conf. Proc.-2003. - 686.-P. 201-210.- (The astrophysics of gravitational wave sources / Ed. J. M. Centrella).

53. Nitadori K., Makino J. Sixth- and eighth-order Hermite integrator for N-body simulations // New Astronomy.-2008.-13.-P. 498-507.

54. Ohta T., Okamura H., Kimura T., et al. Physically acceptable solution of Einstein's equation for many-body system // Progr. Theor. Phys._-1973._-50._P. 492 - 514.

55. Ohta T., Okamura H., Kimura T., et al. Coordinate condition and higher order gravitational potential in canonical formalism // Progr. Theor. Phys._1974._51.P. 1598-1612.

56. Ohta T., Okamura H., Kimura T., et al. Higher order gravitational potential for manybody system // Progr. Theor. Phys.-1974._51.-P. 1220-1238.

57. Overzier R. A., Heckman T. M., Kauffmann G., et al. Hubble space telescope morphologies of local Lyman break galaxy analogs. I. Evidence for starbursts triggered by merging // Astrophys. J._-2008.-677.-P. 37-62.

58. Peters $P$. C. Gravitational radiation and the motion of two point masses // Phys. Rev.-1964.-136.-P. 1224-1232.

59. Peters P. C., Mathews J. Gravitational radiation from point masses in a Keplerian orbit // Phys. Rev.-1963.-131.-P. 435-440.

60. Preto M., Berentzen I., Berczik P., et al. Fast coalescence of massive black hole binaries from mergers of galactic nuclei: Implications for lowfrequency gravitational-wave astrophysics // Astrophys. J._-2011._732.-6 p.

61. Rodríguez Zaurín J., Tadhunter C. N., González Delgado R. M. The properties of the 
stellar populations in ULIRGs. II. Star formation histories and evolution // Mon. Notic. Roy. Astron. Soc.-2010.-403.-P. 1317-1330.

62. Sathyaprakash B. S., Schutz B. F. Physics, astrophysics and cosmology with gravitational waves // Liv. Revs Relativity.-2009._12._-141 p.

63. Sijacki D., Vogelsberger M., Genel S., et al. The Illustris simulation: the evolving population of black holes across cosmic time // Mon. Notic. Roy. Astron. Soc.-2015. 452.-P. 575-596.

64. Silk J., Rees M. J. Quasars and galaxy formation // Astron. and Astrophys.-1998. -331.-P. 1-4.

65. Soffel M. H. Relativity in astrometry, celestial mechanics and geodesy. - Berlin, Heidelberg, New York: Springer-Verlag, 1989.-208 p.

66. Tagoshi H., Ohashi A., Owen B. J. Gravitational field and equations of motion of spinning compact binaries to 2.5 post-Newtonian order // Phys. Rev. D._-2001._63, N 4.- 14 p.

67. van Dokkum $P$. G. The recent and continuing assembly of field elliptical galaxies by red mergers // Astrophys. J.-2005.-130.-P. 2647-2665.

68. Wang L., Berczik P., Spurzem R., et al. The link between ejected stars, hardening and eccentricity growth of super massive black holes in galactic nuclei // Astrophys. J.2014.-780.-14 p.

69. Yagi K., Seto N. Detector configuration of DECIGO/BBO and identification of cosmological neutron-star binaries // Phys. Rev. D.-2011.-83, N 4.-14 p.

70. Zhong S., Berczik P., Spurzem R. Super massive black hole in galactic nuclei with tidal disruption of stars // Astrophys. J.-2014._-792.-17 p.

71. Zinchenko I. A., Berczik P., Grebel E. K., et al. On the influence of minor mergers on the radial abundance gradient in disks of milky-way-like galaxies // Astrophys. J.2015. -806. - 17 p.

Received 01.04.16 